\title{
Effect of transcranial direct current stimulation on post-stroke fatigue
}

\author{
William De Doncker ${ }^{1}\left[\right.$ Sasha Ondobaka ${ }^{1,2} \cdot$ Annapoorna Kuppuswamy $^{1}$
}

Received: 7 January 2021 / Revised: 1 February 2021 / Accepted: 2 February 2021 / Published online: 17 February 2021

(c) The Author(s) 2021

\begin{abstract}
Background Fatigue is one of the most commonly reported symptoms post-stroke, which has a severe impact on the quality of life. Post-stroke fatigue is associated with reduced motor cortical excitability, specifically of the affected hemisphere. Objective The aim of this exploratory study was to assess whether fatigue symptoms can be reduced by increasing cortical excitability using anodal transcranial direct current stimulation (tDCS).

Methods In this sham-controlled, double-blind intervention study, tDCS was applied bilaterally over the primary motor cortex in a single session in thirty stroke survivors with high severity of fatigue. A questionnaire-based measure of trait fatigue (primary outcome) was obtained before, after a week and 5 weeks post stimulation. Secondary outcome measures of state fatigue, motor cortex neurophysiology and perceived effort were also assessed pre, immediately post, a week and 5 weeks post stimulation.

Results Anodal tDCS significantly improved fatigue symptoms a week after real stimulation when compared to sham stimulation. There was also a significant change in motor cortex neurophysiology of the affected hemisphere and perceived effort, a week after stimulation. The degree of improvement in fatigue was associated with baseline anxiety levels.

Conclusion A single session of anodal tDCS improves fatigue symptoms with the effect lasting up to a week post stimulation. tDCS may therefore be a useful tool for managing fatigue symptoms post-stroke.

Trial registration NCT04634864

Date of registration 17/11/2020-“retrospectively registered".

Keywords tDCS $\cdot$ Effort $\cdot$ Fatigue $\cdot$ Perception $\cdot$ Stroke

$\begin{array}{ll}\text { Abbreviations } \\ \text { PSF } & \text { Post-stroke fatigue } \\ \text { TMS } & \text { Transcranial Magnetic stimulation } \\ \text { M1 } & \text { Primary motor cortex } \\ \text { PE } & \text { Perceived effort } \\ \text { tDCS } & \text { Transcranial direct current stimulation } \\ \text { IO } & \text { Recruitment curve slope } \\ \text { FSS-7 } & \text { Fatigue Severity Scale-7 } \\ \text { HADS } & \text { Hospital Anxiety Depression Scale } \\ \text { VAS } & \text { Visual Analogue Scale } \\ \text { EMG } & \text { Electromyogram } \\ \text { FDI } & \text { First Doral interosseous }\end{array}$

\begin{tabular}{|c|c|}
\hline RMT & Resting motor threshold \\
\hline MEP & Motor evoked potential \\
\hline RMT-A & $\begin{array}{l}\text { Resting motor threshold of affected } \\
\text { hemisphere }\end{array}$ \\
\hline $\mathrm{IO}_{\text {Slope }^{-\mathrm{A}}}$ & $\begin{array}{l}\text { Recruitment curve slope of affected } \\
\text { hemisphere }\end{array}$ \\
\hline RMT-U & $\begin{array}{l}\text { Resting motor threshold of Un-affected } \\
\text { Hemisphere }\end{array}$ \\
\hline $\mathrm{IO}_{\text {Slope }^{-U}}$ & $\begin{array}{l}\text { Recruitment curve slope of Un-affected } \\
\text { Hemisphere }\end{array}$ \\
\hline GABA & $\gamma$-Aminobutiric acid \\
\hline
\end{tabular}
\end{abstract}

William De Doncker

William.doncker.11@ucl.ac.uk

1 Department of Clinical and Movement Neurosciences, Institute of Neurology, University College London, London WC1N 3BG, United Kingdom

2 Institute of Cognitive Neuroscience, University College London, London WC1N 3BG, United Kingdom

\section{Introduction}

Debilitating fatigue that persists for months and sometimes years after stroke is relatively common in stroke survivors with a prevalence as high as $70 \%[1,2]$. Post-stroke fatigue (PSF) has been identified as the top unmet need among 
stroke survivors living in the community and is a top priority for further research [3-5].

Despite the high prevalence of fatigue, the pathophysiology of chronic fatigue is poorly understood with little evidence-based therapy to alleviate fatigue [6]. Our recent work aimed at understanding the underlying neurophysiology of PSF provided a potential target for modulation that may reduce the symptoms of PSF. We showed using transcranial magnetic stimulation (TMS), that cortical excitability at rest, specifically the primary motor cortex (M1) of the affected hemisphere, is reduced in those who report high levels of PSF [7]. Motor cortex excitability as measured by TMS is normally associated with the motor function of the targeted muscle [8]. However, TMS measures of motor cortex excitability have also been associated with non-motor functions such as perception and attention [9, 10]. Given the homogeneity of motor function in the investigated stroke cohort, we argued that reduced motor cortex excitability was a reflection of altered perceptual processing in relation to muscle contraction i.e. altered effort perception.

Perceived effort (PE) is heavily influenced by both expectations and feedback, efferent and afferent input. The active inference framework of sensorimotor control provides a simple framework that integrates both efferent and afferent input to explain motor control [11-19]. Within this framework, expectations set the gain for afferent input and PE is a psychophysical output of the gain function [20]. We recently showed that those with greater PSF did indeed show greater PE [21]. Increased PE may be driven by altered gain, as measured by motor cortex excitability [20]. We hypothesised that altering the gain i.e. increasing motor cortex excitability, will reduce $\mathrm{PE}$ and subsequently reduce fatigue symptoms.

Transcranial direct current stimulation (tDCS) is a non-invasive brain stimulation technique that increases or decreases cortical excitability when applied to the M1, depending on the montage and stimulation parameters used $[22,23]$. tDCS has promising potential therapeutic applications due to its ease of use, low cost and lack of physiological and behavioural side effects [24, 25]. A single session of tDCS for a few minutes can result in after effects that last for more than an hour, with within-session repeated tDCS resulting in more pronounced and longer-lasting effects [26, 27]. tDCS modulates cortical excitability in stroke survivors and has been widely used in the treatment of various neurological and psychiatric disorders, including the treatment of multiple sclerosis fatigue [28-37]. tDCS has also been used to enhance sport performance and has been shown to reduce rate of perceived exertion in healthy individuals [38].

The primary aim of this exploratory study was to use anodal tDCS over the M1 to reduce PSF. The secondary aim was to investigate the potential mechanisms that underlie the hypothesised effect on PSF.

\section{Methods}

\section{Study design}

This was a double-blind, sham-controlled study with a single session of bilateral anodal tDCS chosen as the method of intervention. Patients visited the laboratory on three separate occasions, with tDCS applied only on the first visit. The second visit took place 1 week later with the third visit taking place one month after visit two and will be termed week and month throughout the manuscript (Fig. 1). The primary outcome measure was a change in trait fatigue. Secondary outcome measures included state fatigue, explicit and implicit measures of PE and motor cortex physiology measures of resting motor thresholds (RMT) and slope of recruitment curves $\left(\mathrm{IO}_{\text {Slope }}\right)$ of the affected and un-affected hemisphere assessed using TMS. The primary outcome measure was recorded at three distinct time points (pre stimulation, week and month). All other outcome measures were recorded at four distinct time points (pre stimulation, immediately post stimulation, week and month).

\section{Subjects}

The study was approved by London Bromley Research Ethics Committee (16/LO/0714). Stroke survivors were recruited via Clinical Research Network at University
Fig. 1 Study Design indicating the sequence in which procedures were done for each of the sessions at the 4 different time points (pre tDCS, immediately post-tDCS, week and month time points). PE perceived effort; TMS transcranial magnetic stimulation; RMT resting motor threshold; IO recruitment curves; tDCS transcranial direct current stimulation

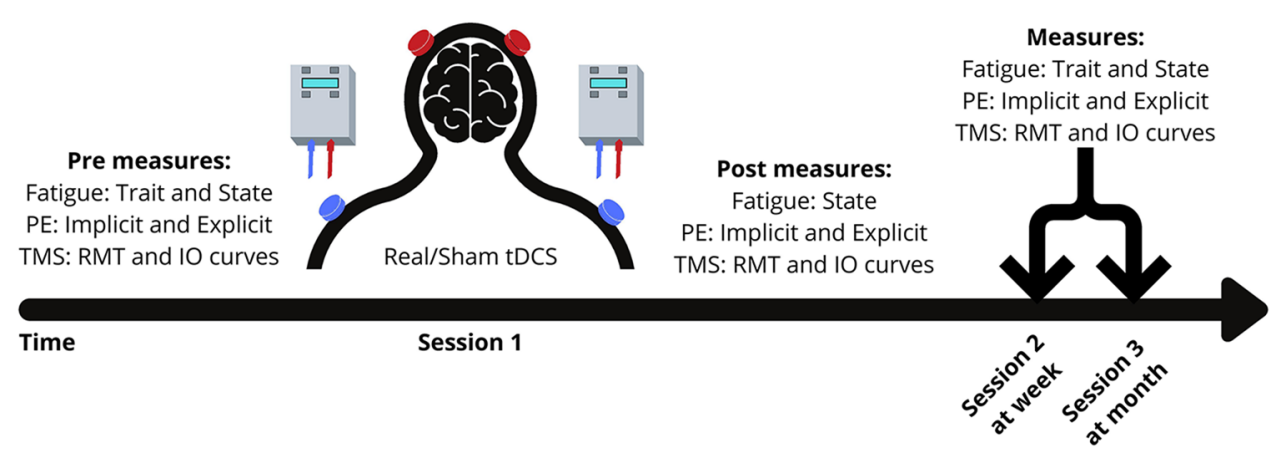


College NHS Trust Hospital, a departmental Stroke Database and from the community.

Inclusion criteria: Date of stroke $>3$ months, first-time stroke, age $\geq 18$ years; Fatigue Severity Scale (FSS-7) $\geq 4$. A score of $\geq 4$ on FSS-7 indicates the presence of clinically significant fatigue [39]. Exclusion criteria: use of centrallyacting medication which may affect the level of fatigue, depression, and anxiety; depression scores $\geq 11$ (Hospital Anxiety and Depression Scale-HADS); grip strength and manual dexterity (nine-hole peg test) $\leq 60 \%$ of unaffected hand.

The minimal clinically important difference on the FSS-7 is 0.45 , with differences greater than 0.45 predicting a significant effect on the quality of life $[40,41]$. To detect the minimal clinically important difference in fatigue with $80 \%$ power $(0.80)$ and a significance level alpha of 0.05 , a sample size of 11 subjects per group is needed. Twice the number of patients were allocated to the real stimulation group than necessary, as previous studies using tDCS in multiple sclerosis fatigue and healthy individuals showed that approximately $50 \%$ of patients respond to tDCS [31, 42]. Thirty-three patients were recruited into the study and were randomly allocated to the real $(n=22)$ or sham $(n=11)$ stimulation groups (Fig. 2). All patients gave written informed consent in accordance with the Declaration of Helsinki. Patient demographics for both groups are found in Table 1.

A

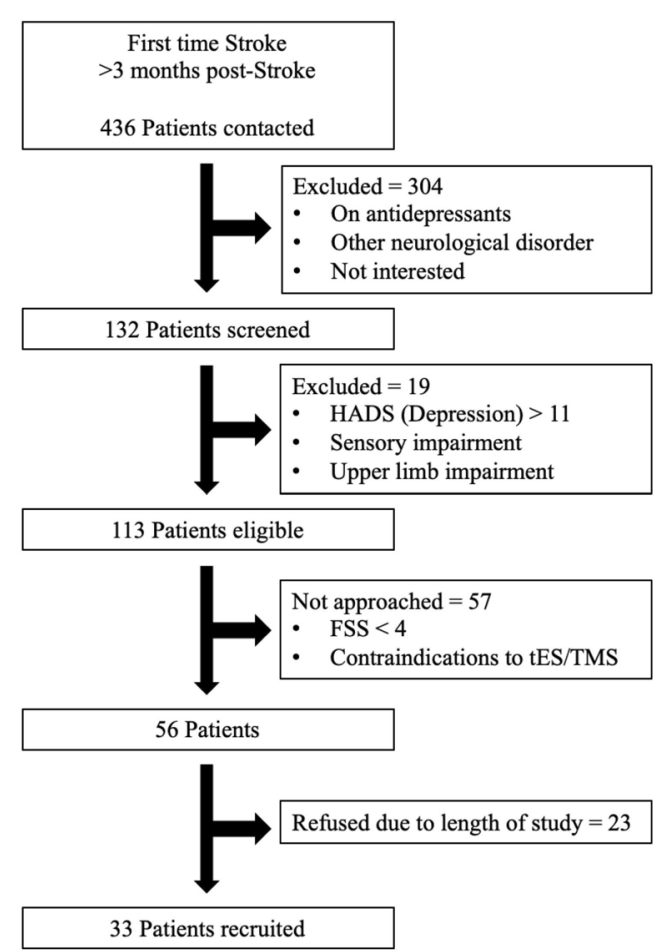

Table 1 Patient demographics and clinical data for the real and sham stimulation groups

\begin{tabular}{lll}
\hline & Real $(N=20)$ & Sham $(N=10)$ \\
\hline Age (years) & $56.95(13.17)$ & $59.83(11.66)$ \\
Time since stroke (years) & $4.19(5.43)$ & $4.83(6.47)$ \\
FSS-7 & $5.84(0.63)$ & $5.14(0.74)$ \\
HADS-Depression & $6.15(3.31)$ & $6.20(3.39)$ \\
HADS-Anxiety & $5.80(2.78)$ & $7.10(4.31)$ \\
Grip (\% unaffected hand) & $85.83(22.09)$ & $80.00(29.32)$ \\
NHPT (\% unaffected hand) & $85.14(27.91)$ & $77.37(23.36)$ \\
Gender & & \\
Females I Males & $11 \mid 9$ & $4 \mid 6$ \\
Hemisphere Affected & & $4 \mid 6$ \\
Left I Right & $10 \mid 10$ & 8 | \\
Type of stroke & & 1 \\
Ischaemic I Haemorrhagic & $16 \mid 4$ & 5 \\
Lesion location territory & & 0 \\
ACA & 1 & 4 \\
MCA & 16 & 10 | 0 \\
PCA & 2 & \\
Brainstem and cerebellum & 1 & \\
Dominant hand & $19 \mid 1$ & \\
Right I Left & & \\
\hline
\end{tabular}

FSS-7 Fatigue severity Scale-7; HADS Hospital Anxiety and Depression Scale; NHPT Nine-hole peg test; $A C A$ Anterior cerebral artery; $M C A$ Middle cerebral artery; $P C A$ Posterior cerebral artery

B

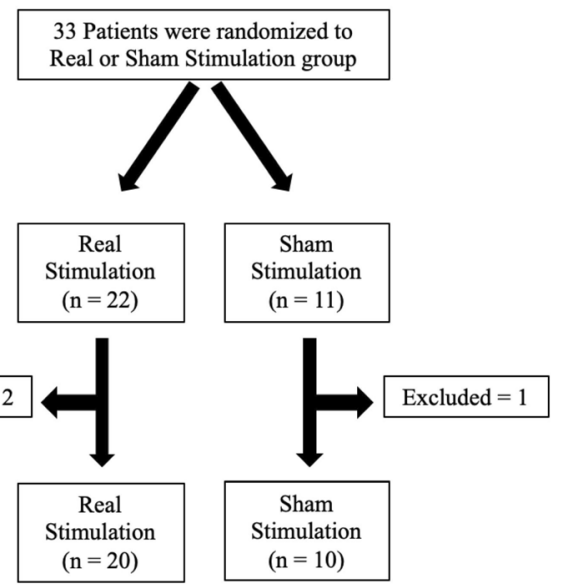

Fig. 2 a Study recruitment and b randomisation (HADS Hospital Anxiety and Depression Scale; FSS Fatigue Severity Scale; $t E S$ transcranial Electrical Stimulation; TMS Transcranial magnetic stimulation) 


\section{Questionnaires}

Trait and state measures of fatigue were captured during the study. Trait fatigue represents the experience and impact of fatigue on day to day living for a pre-determined time period leading up to the day of testing, whereas state fatigue characterizes fatigue at a given moment in time. Trait fatigue was quantified using the FSS-7, a seven-item questionnaire asking for ratings of fatigue ranging from one to seven (strongly disagree to strongly agree) over the preceding week from the day of administration [43]. An average score of one indicates no fatigue while an average score of seven indicates very severe fatigue. State fatigue was quantified using a visual analogue scale (VAS) ranging from zero to ten (Not at all tired to extremely tired). Patients also completed the HADS, a 14-item questionnaire with a depression and anxiety subscale, prior to the stimulation. A score of 0 to 7 for either subscale could be regarded as being in the normal range, with a score of 11 or higher indicating the probable presence of the mood disorder [44].

\section{Stimulation}

tDCS was applied using two battery-driven stimulators (DCStimulator Plus, NeuroConn, Germany) while patients were awake and at rest. Four $35 \mathrm{~cm}^{2}$ rubber electrodes coated with conductive paste were secured with self-adhesive bandages. The anode of each stimulator was placed over the left and right M1 (C3 and C4 according to the 10-20 EEG system), while the cathode of each stimulator was placed over the ipsilateral left and right shoulders. This tDCS montage has previously been shown to reduce the perception of effort and increase corticospinal excitability in healthy individuals [38]. Real tDCS involved two 20-min sessions of stimulation at $2 \mathrm{~mA}$ separated by a 10-min break in between. The current was ramped up for $30 \mathrm{~s}$ until reaching $2 \mathrm{~mA}$ and ramped down for $30 \mathrm{~s}$ at the end of the stimulation period. Stimulation intensity and duration complied with current safety recommendations [45]. For sham stimulation, the current was ramped down immediately after ramping up, providing effective blinding [46]. The patient and researchers were blind to the applied stimulation (real or sham). At the end of stimulation, patients were explicitly asked whether they thought they received real or sham stimulation.

\section{Perceived effort}

PE was measured in an isometric handgrip task with a hand-held dynamometer (Biometrics Ltd, Newport, UK) performed using the dominant hand [21]. Force data from the dynamometer were acquired at $500 \mathrm{~Hz}$ via a data acquisition interface (Power1401, CED) and recorded in MATLAB (2016b, MathWorks). Each trial was 5 s long, in which patients were required to sustain a grip force for $3 \mathrm{~s}$ at $20 \%$, $40 \%$, or $60 \%$ of their maximum voluntary force. Immediate force feedback was shown on the monitor as a filling of a red bar, which turned green once the minimal required target force, indicated by a cross on the screen, was reached. The grip force-visual feedback relationship was individually adjusted for every patient to eliminate potential influence on PE. Before the experiment, patients practiced each force level with their dominant hand to familiarize themselves with the effort required and performed a line familiarization. In the line familiarization, patients were shown 3 "short' lines (1, 2, and $3 \mathrm{~cm}$ ), and 3 "long" lines (10, 11, and $12 \mathrm{~cm})$. After the presentation of the 6 lines, patients were shown each of the learned lines without information about the category it belonged to and were asked to judge the line length. Patients responded using the keyboard: left arrow key for "short" and right arrow key for "long". They were then asked to rate their confidence in their response using a VAS. If patients' response was $<100 \%$ correct, the procedure was repeated until they were able to distinguish between short and long lines.

During the PE task, each grip was followed by a line length estimation. The line presented could have a length of $3.5-8.5 \mathrm{~cm}$ with a total of 24 different line lengths, 12 short and 12 long. Twenty-four lines presented under the 3 force conditions resulted in a total of 72 trials divided into 3 blocks. The order of forces and line lengths was randomized with equal numbers of the 3 different force levels in each block. Participants reported if the presented line was short or long based on the length of lines presented during the familiarization phase. If they determined the presented line to be shorter than half the length of the longest line presented during the familiarization $(12 \mathrm{~cm})$, they reported short; otherwise, they reported long. These blocks were used as an implicit measure of PE.

After 3 blocks, participants performed a final block of 9 trials. This block was used as an explicit measure of PE. Each trial consisted of a 5-s grip with visual feedback at the 3 different force levels, $20 \%$, $40 \%$, or $60 \%$ of maximum voluntary force, with 3 trials for each force level. This was followed by the question, "How effortful was the squeeze?" Patients had to respond using a VAS ranging from "not at all" to "very hard."

\section{Surface electromyogram and TMS}

Electromyogram (EMG) recordings were obtained from the first dorsal interosseous (FDI) muscle using surface electrodes (1041PTS Neonatal Electrode, Kendell) in a belly-tendon montage with the ground positioned over the flexor retinaculum of the hand. The signal was amplified with a gain of 1000 (D360, Digitmer, Welwyn Garden City, UK), bandpass filtered (100-1000 Hz), digitized at $10 \mathrm{kHz}$ 
(Power1401, CED, Cambridge, UK) and recorded with Signal version 6.04 software (CED, Cambridge, UK).

TMS (figure-of-eight coil with wing diameter, $70 \mathrm{~mm}$; Magstim 2002, Magstim, Whitland, UK) was used to stimulate the hand area of the M1. The coil was held tangentially on the scalp at $45^{\circ}$ to the mid-sagittal plane to induce a posterior-anterior current across the central sulcus. The subjects were instructed to stay relaxed with their eyes open and their legs uncrossed. The motor 'hotspot' of the FDI muscle was determined as described previously [7]. RMT was defined as the lowest intensity required to evoke a motor evoked potential (MEP) at the hotspot of at least $50 \mu \mathrm{V}$ in a minimum of 5 of 10 consecutive trials while subjects were at rest. IO curves were acquired at rest at the hotspot using TMS intensities set at 100,110,120,130,140 and $150 \%$ of RMT. Six pulses at each of the 6 intensities were delivered in a randomized order with an inter-trial interval of $4 \mathrm{~s}$, giving thirty-six trials in total. This procedure was repeated for both

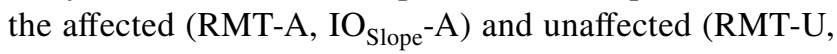
$\left.\mathrm{IO}_{\text {Slope }}-\mathrm{U}\right)$ hemispheres.

\section{Analysis of questionnaires}

The FSS-7 score was calculated by averaging all items for each of the three-time points.

The total score was taken for the anxiety and depression subscales of HADS, HADS-Anxiety and HADS-Depression respectively, and were considered as independent measures.

\section{TMS analysis}

The data were analysed using custom-written routines in Matlab (2018a, Mathworks). Peak-to-peak MEP amplitudes for each condition were estimated from the EMG recordings. All trials were visually inspected and approximately $7 \%$ of trials with pre-contraction and size $\leq 0.025 \mathrm{mV}$ were excluded across all participants. A linear fit was applied to all the MEP data across the six conditions (100-150\% RMT) for each participant at each session. The quality of the linear fit was evaluated by calculating the r-squared value for each participant across each session. The grand mean r-squared value across all individuals and all session and the resulting standard deviation $\left(R^{2}=0.86 \pm 0.12\right)$ demonstrate an overall good fit of the MEP data with little variability within conditions. The gradient of the linear fit was subsequently calculated for each participant in each of the four sessions and for each hemisphere (affected and un-affected hemisphere) giving us the slope of the recruitment curve.

\section{PE analysis}

To obtain a measure of explicit PE, VAS scores were averaged across all trials in each force level in each individual.
As there was no difference across force level, the average VAS score across all force levels was used as an explicit measure of PE for each of the four-time points. To obtain a measure of implicit PE, the sum of the number of lines reported as long for each individual in each force level was calculated. As there was no difference across force level, the average number of lines across all force levels was used as an implicit measure of PE for each of the four-time points.

\section{Statistical analysis}

All statistical analysis was performed using R (RStudio Version 1.2.5033). Assumptions of a normal distribution of the primary and all secondary outcome variables were assessed using the Shapiro-Wilk test. All data were nonnormally distributed $(p<0.05)$. To test for changes over time, a non-parametric Friedman test was performed for the primary outcome variable (trait fatigue) and all secondary outcome variables (state fatigue, RMT-A, RMT$\mathrm{U}, \mathrm{IO}_{\text {Slope }}-\mathrm{A}, \mathrm{IO}_{\text {Slope }}-\mathrm{U}, \mathrm{PE}-\mathrm{implicit}$ and PE-explicit), separately for the sham and real intervention groups as in Saiote et al. [31]. When significant results were found, pairwise comparison between baseline and each postmeasurement day were performed using Wilcoxon signedrank test. To analyse the effect of real stimulation versus sham stimulation across both primary and secondary outcome measures, the changes in scores were calculated by normalising each day to baseline (pre stimulation) and then compared within each day using a Wilcoxon signedrank test. Adjustment for multiple comparisons was performed using Bonferroni correction.

A spearman correlation was used to examine the association between baseline trait fatigue scores and the change in trait fatigue a week after stimulation in both the sham and real stimulation groups. To identify the potential mechanisms that drive the change in trait fatigue in the real stimulation group a multiple linear regression was used with demographic data and secondary outcome variables that were significantly different between the real and sham stimulation groups used as predictors. Collinearity amongst the predictors used in the multiple regression model was assessed by computing the variance inflation factor (VIF). No VIF value exceeded a score of 5, demonstrating that there was no collinearity amongst the predictors used. Goodness of fit was assessed using the BIC (lower BIC indicates a better fitting model) to identify the combination of variables that best predicted the outcome variable, the change in trait fatigue. Assumptions of normality and homoscedasticity of the residuals for each model were assessed visually using quantile-quantile normal plots and fitted- versus residualvalue plots. 


\section{Results}

Two patients from the real stimulation group decided to withdraw from the study during visit 1 as they found the stimulation uncomfortable and one patient from the sham stimulation group was excluded because they started taking antidepressants after session 1. No serious adverse events were reported. Thirty patients were included in the final analysis, with twenty in the real stimulation group and ten in the sham stimulation group.

\section{Trait and state fatigue}

The Friedman test showed a significant effect of time on trait fatigue in the real stimulation group $\left(\chi^{2}(2)=15.5 ; p<0.001\right)$ but not in the sham stimulation group $\left(\chi^{2}(2)=0.154\right.$; $p=0.926)$. Post-hoc analysis with Wilcoxon signed-rank tests showed that FSS-7 scores decreased compared to baseline both at the week $(V=198, Z=0.777, p=0.002)$ and month $(V=142, Z=0.535, p=0.047)$ time point in the real stimulation group. FSS-7 at the week time point was also lower than at the month time point $(V=21, Z=0.639$, $p=0.016$ ). Post-hoc Wilcoxon signed-rank test of the normalised FSS-7 scores revealed a significant difference between the sham and real stimulation group at the week time point ( $W=52.5, Z=0.382, p=0.0386$ ) but not at the month time point $(\mathrm{W}=65.5, \mathrm{Z}=0.277, \mathrm{p}=0.134)$, seen in Fig. 3a.

The Friedman test showed no significant effect of time on state fatigue in the real $\left(\chi^{2}(3)=0.97 ; p=0.809\right)$ or sham $\left(\chi^{2}(3)=3 ; p=0.392\right)$ stimulation group. Post-hoc Wilcoxon singed-rank test for the normalised state fatigue scores revealed no significant difference between the sham and real stimulation group at any time point (Fig. 3b).

\section{Neurophysiology}

The Friedman test showed a significant effect of time on $\mathrm{IO}_{\text {Slope }} \mathrm{A}$ in the real stimulation group $\left(\chi^{2}(3)=11.2\right.$, $p=0.0106)$ but not in the sham stimulation group $\left(\chi^{2}(3)=0.75\right.$, $p=0.861)$. Post-hoc analysis with Wilcoxon signed-rank tests showed that $\mathrm{IO}_{\text {Slope }}-\mathrm{A}$ decreased compared to baseline at the week ( $V=96, Z=0.730, p=0.024)$ but not at the immediate post and month time point in the real stimulation group. Posthoc Wilcoxon signed-rank test of the normalised $\mathrm{IO}_{\text {Slope }} \mathrm{A}^{-\mathrm{A}}$ scores revealed a significant difference between the sham and real stimulation group at the week time point $(W=23$, $Z=0.480, p=0.024$ ) but not at the immediate post and month time point. The Friedman test showed no significant effect of time on RMT-A in the real $\left(\chi^{2}(3)=2.62, p=0.454\right)$ or sham $\left(\chi^{2}(3)=2.06, p=0.560\right)$ stimulation groups. Post-hoc
Wilcoxon singed-rank test for the normalised RMT-A revealed no significant difference between the sham and real stimulation group at any time point.

The Friedman test showed no significant effect of time on RMT-U or $\mathrm{IO}_{\text {Slope }}-\mathrm{U}$ in the real $\left(\chi^{2}(3) 2.71, p=0.438\right.$; $\left.\chi^{2}(3)=3.38, \mathrm{p}=0.337\right)$ or sham $\left(\chi^{2}(3)=5.62, p=0.132\right.$; $\left.\chi^{2}(3)=2.2, p=0.552\right)$ stimulation groups. Post-hoc Wilcoxon singed-rank test for the normalised RMT-U and $\mathrm{IO}_{\text {Slope }} \mathrm{U}$ scores revealed no significant difference between the sham and real stimulation group at any time point (Fig. 3c-f).

\section{Perceived effort}

The Friedman test showed no significant effect of time on PE-implicit or PE-explicit in the real $\left(\chi^{2}(3)=5.60, p=0.905\right.$; $\left.\chi^{2}(3)=0.2, p=0.978\right)$ or sham $\left(\chi^{2}(3)=4.24, p=0.237\right.$; $\left.\chi^{2}(3)=6.73, p=0.0809\right)$ stimulation groups. Post-hoc Wilcoxon singed-rank test for the normalised PE-explicit scores revealed no significant difference between the sham and real stimulation group at any time point. Post-hoc Wilcoxon singed-rank test for the normalised PE-implicit scores revealed a significant difference between the sham and real stimulation group at the week time point $(W=39.5$, $Z=0.391, p=0.0491)$ but not at the immediate post and month time point (Fig. $3 \mathrm{~g}-\mathrm{h}$ ).

\section{Change in trait fatigue}

There was no association between baseline FSS-7 scores and the change in FSS-7 score at the week time point in the real $\left(r_{\mathrm{s}}=-0.2, p=0.39\right)$ and sham $\left(r_{\mathrm{s}}=0.2\right.$, $p=0.57$ ) stimulation group (Fig. 4a). The multiple linear regression model with normalised $\mathrm{IO}_{\text {Slope }} \mathrm{A}$ at the week time point and baseline HADS-Anxiety scores as predictors was the best fitting model $(\triangle \mathrm{FSS}$ week $=$ $-2.775+0.436 \Delta \mathrm{IO}_{\text {Slope }}-\mathrm{A}+0.285 \mathrm{HADS}$-Anxiety). A significant regression equation was found $(F(2,13)=5.345$, $p=0.020$ ), with an adjusted $R^{2}$ of 0.37 . Baseline HADSAnxiety significantly explained the change in FSS-7 scores $(t=2.925, p=0.013)$, whereas normalised $\mathrm{IO}_{\text {Slope }}-\mathrm{A}$ at the week time point $(t=1.760, p=0.104)$ was not a significant predictor of the change in FSS-7 scores (Fig. 4b). Beta coefficients of the predictors used in the model together with their associated $95 \%$ confidence intervals and $\mathrm{p}$-values are found in Table 2.

\section{Discussion}

In this study, we aimed to improve fatigue symptoms in minimally impaired, non-depressed stroke survivors using bilateral anodal tDCS over the M1. We show a significant 

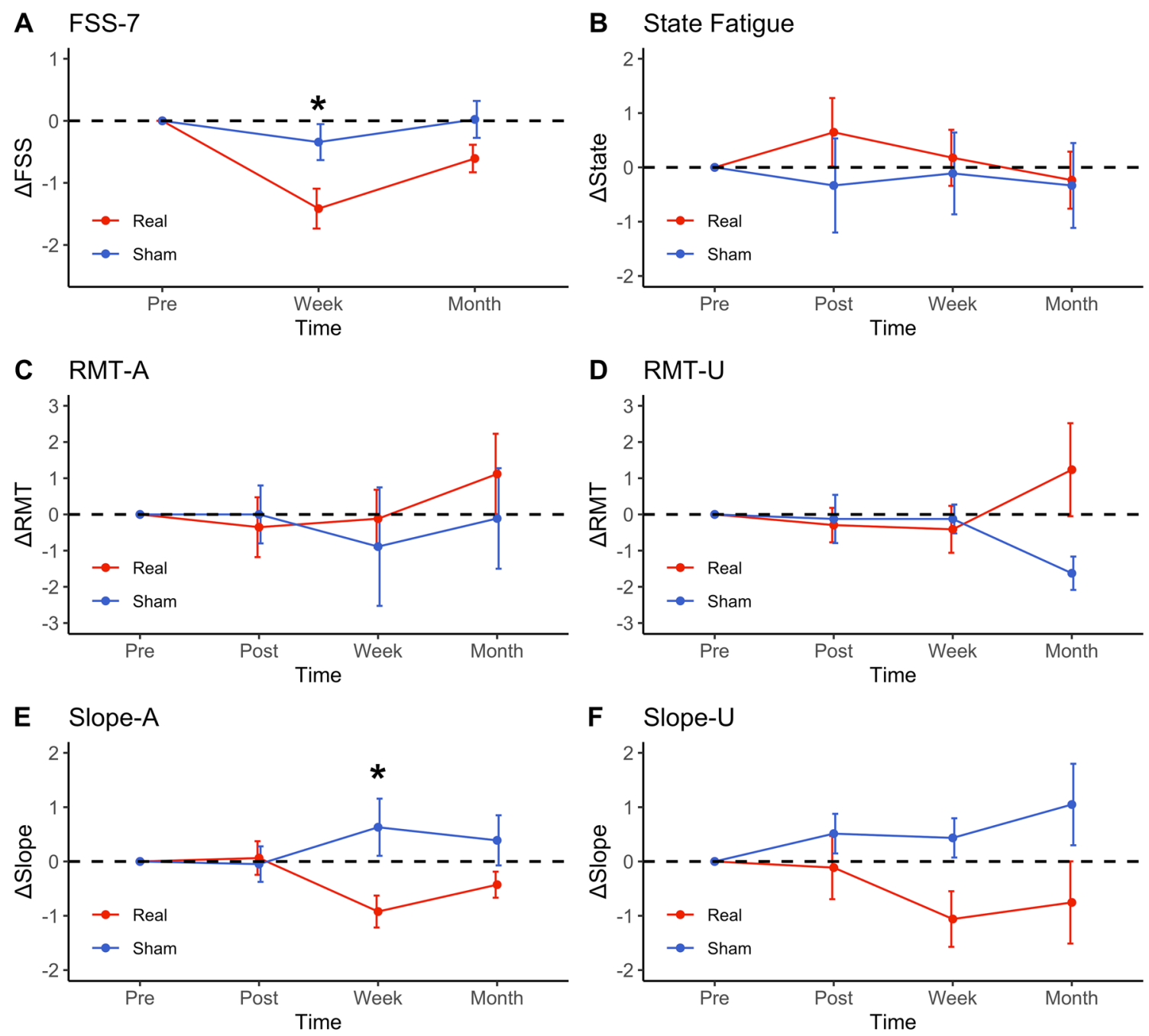

F Slope-U
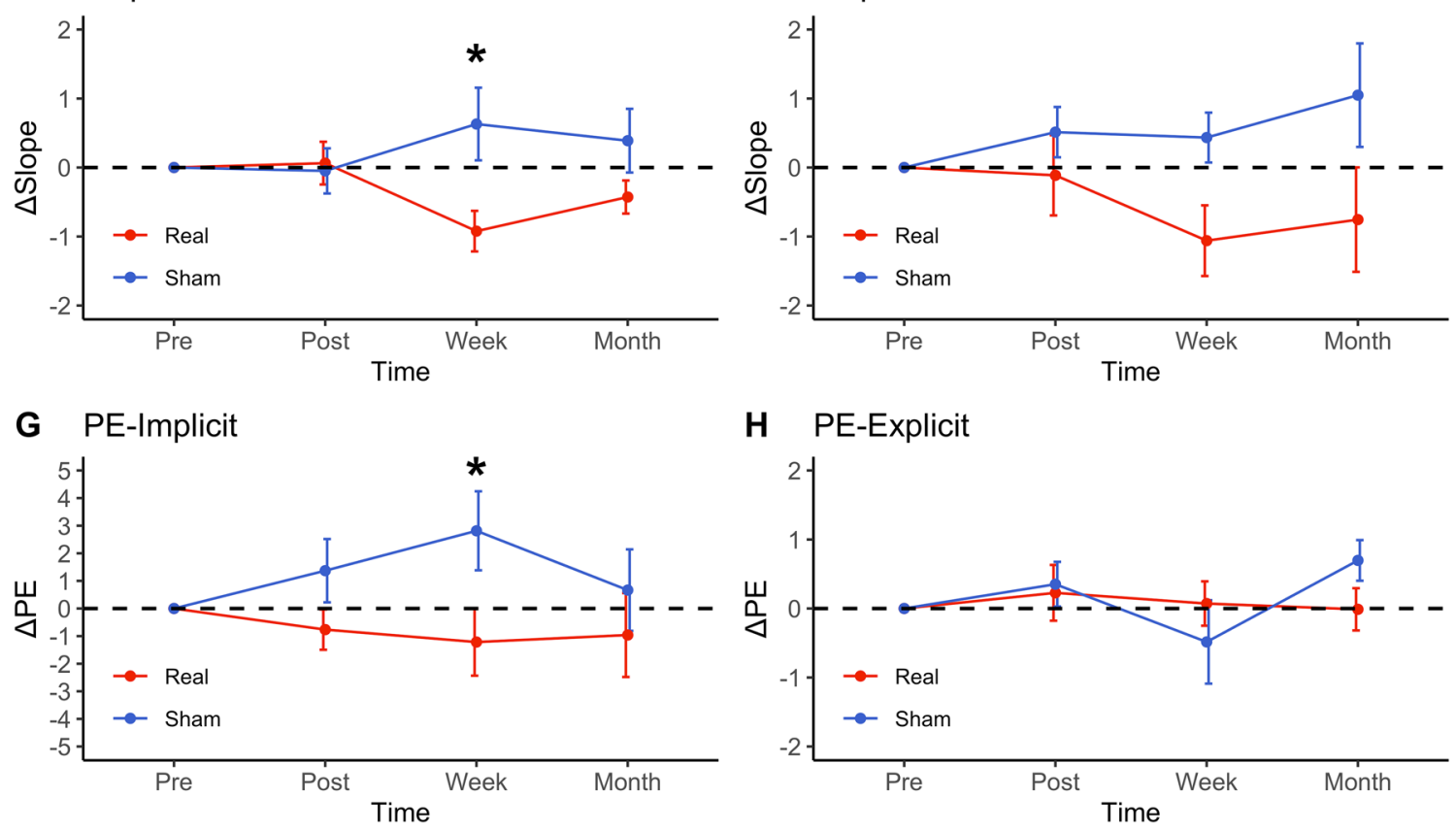

Fig. 3 Changes in trait fatigue (a), state fatigue (b), resting motor threshold of the affected and unaffected hemisphere (c, d), slope of the recruitment curve of the affected and unaffected hemisphere (e, f), implicit perceived effort (g) and explicit perceived effort $(\mathrm{H})$ compared to baseline (pre-stimulation time point) across the different time points for both the real (red) and sham (blue) stimulation group.

reduction in trait fatigue a week following anodal tDCS. There were also significant differences between the real and sham stimulation groups in $\mathrm{IO}_{\text {Slope }}$ of the affected hemisphere and implicit measures of PE a week after

Error bars represent standard error of the means. Significance levels are indicated by $*(p<0.05)$. FSS-7 $=$ Fatigue Severity Scale-7; RMT$A=$ Resting motor threshold of affected hemisphere; $R M T$-U $=$ Resting Motor threshold of Un-affected hemisphere; $I O_{\text {Slope }}-A$ recruitment curve slope of affected hemisphere; $I O_{\text {Slope }}-U$ recruitment curve slope of Un-affected hemisphere; PE Perceived Effort

stimulation. Those with the greatest drop in trait fatigue a week after anodal tDCS also had the lowest anxiety scores prior to stimulation. 


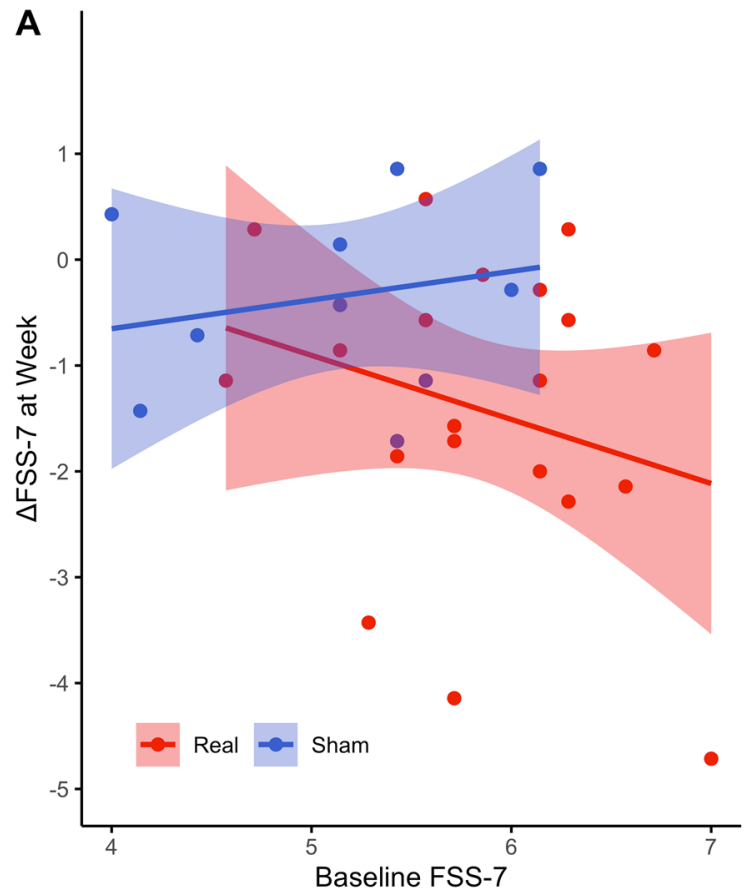

Fig. 4 Correlation between baseline FSS-7 and the change in FSS-7 at the week time point for the real (red) and sham (blue) stimulation groups with the $95 \%$ confidence interval (A). The association between the baseline HADS-Anxiety levels and the change in FSS-7

Table 2 Multiple linear regression results with the change in FSS-7 at the week time point as the outcome variable and change in $\mathrm{IO}_{\text {Slope }}{ }^{-\mathrm{A}}$ and HADS-Anxiety as predictors

\begin{tabular}{lllll}
\hline & Beta coefficient & $2.5 \% \mathrm{CI}$ & $97.5 \% \mathrm{CI}$ & $p$ value \\
\hline (Intercept) & -2.775 & -4.344 & -1.207 & 0.002 \\
$\Delta$ Slope-A & 0.436 & -0.212 & 1.085 & 0.170 \\
HADS-Anxiety & 0.285 & 0.066 & 0.505 & 0.015 \\
\hline
\end{tabular}

Table includes beta coefficients, 95\% confidence intervals and the associated $p$ values for each predictor

FSS-7 Fatigue Severity Scale-7; $I O_{\text {Slope }}-A$ recruitment curve slope of the affected hemisphere; HADS Hospital Anxiety and Depression Scale; $C I$ confidence interval

\section{tDCS and fatigue}

Anodal tDCS increases cortical excitability [28] and reduces the perception of effort in healthy participants during an endurance performance task [38]. The effects of tDCS are not specific to the targeted brain regions but spread to distinct cortical and subcortical structures [47]. A single session of anodal tDCS was expected to increase motor cortex excitability temporarily resulting in reduction of PE by modulating excitability and connectivity of regions upstream of M1 [12, 13, 48]. By resetting PE in a physical task, the carry over effects on behaviour would accumulate eventually

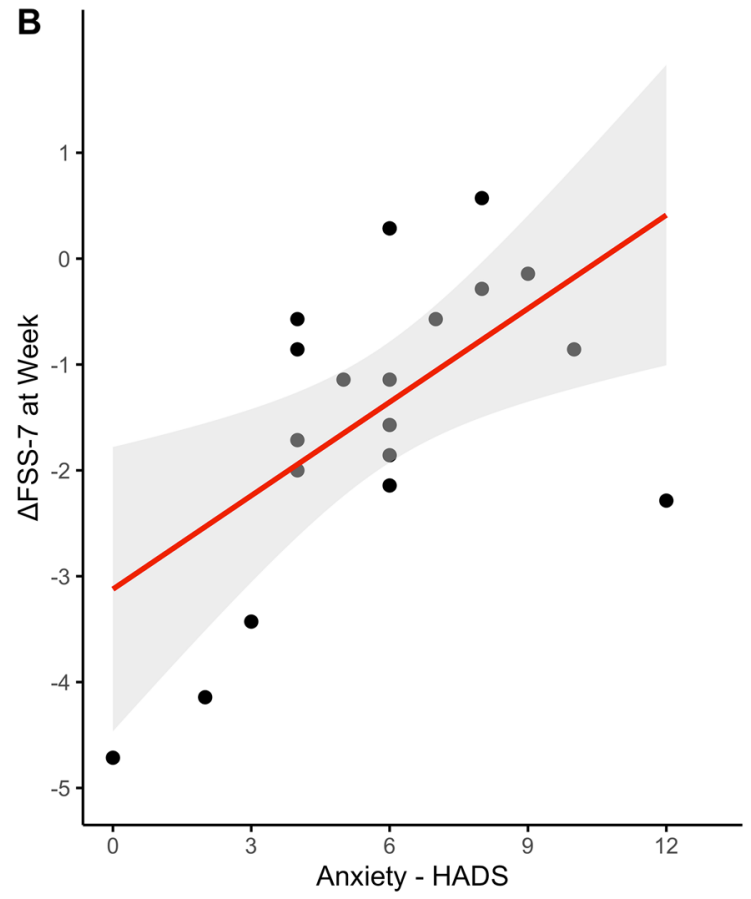

at the week time point for the real stimulation group with its associated 95\% confidence interval (B). FSS-7 Fatigue Severity Scale-7; $H A D S$ Hospital Anxiety and Depression Scale

reducing fatigue levels. This reduction in fatigue levels would be evident by changes in trait fatigue but not necessarily state fatigue. The current results partly support this theory by showing a reduction in trait fatigue a week posttDCS. Fatigue has been identified as one of the top unmet needs for chronic stroke survivors within the community and interferes most with their activities of daily living [3, 4]. Currently, there are no effective interventions for PSF $[49,50]$. The proposed intervention, if confirmed to be effective in a larger and a more heterogeneous cohort of patients, will represent a simple, low-cost and risk-free procedure for reducing fatigue symptoms $[24,30]$.

\section{Neurophysiology}

RMT is a measure of motor corticospinal excitability and anodal tDCS reduces RMT [28]. The lack of effect of tDCS on RMT in this study could have been driven by our split stimulation paradigm of two 20-min periods invoking homeostatic metaplasticity [51], with the second 20-min stimulation reversing the effects of the first $20 \mathrm{~min}$. The slope of IO curves following real tDCS reduced significantly in the affected hemisphere when compared to sham tDCS. IO curves are thought to measure the excitability of pathways upstream of the motor cortex, therefore a measure of inputs to the motor cortex 
$[52,53]$. The slope of IO curves represents the gain of descending corticospinal tract, possibly driven from higher motor areas [54, 55]. However, a reduction and not an increase in slope is puzzling. Steeper IO represent greater recruitment of higher-order motor areas such as the supplementary motor area and premotor cortex [53]. As there are no normative values for IO curves, it is hard to speculate if tDCS induced shallowing of IO curves is a reflection of homeostatic normalisation of inputs to the motor cortex, but offers one possible explanation of the IO results.

\section{Perceived effort}

PE has mostly been tested in post-exercise paradigms where measures such as VAS and Borg Scales have been validated against physiological measures of exertion such as heart rate and maximal aerobic capacity $[56,57]$. They conclude PE is less subject to bias in some populations $[58,59]$. However, in the current study PE is measured in a non-exercise paradigm and in a disease population with a condition that is highly stigmatised and under recognised [60,61]. Hence, we expected to see a response bias in PE. Therefore, in addition to an explicit measure of PE, we introduce a novel implicit measure of PE based on line length perception. This measure takes advantage of the susceptibility of visual perception to physical effort where high effort unfavourably biases distance estimation [62]. On similar lines, a line length estimation task was developed and shown to be biased by prior exertion, which we use as a measure of implicit PE [21, 63]. We showed that trait fatigue is explained by implicit PE but not explicit PE in a physical task [21]. In the current study, we show a significant difference between real and sham stimulation in implicit PE but not in explicit PE a week after anodal tDCS. It could be that implicit, perceived effort is the first to respond to tDCS and if the effects were maintained, would result in a reduction in explicit PE. A second possible explanation is that reducing implicit PE that is sufficient to alleviate fatigue. This difference appears to be primarily driven by an increase in implicit PE in the sham stimulation group. Perhaps in the real stimulation group this task-specific increase in $\mathrm{PE}$ was suppressed. The test-retest reproducibility of the paradigm used to measure implicit PE has not been examined in the absence of tDCS, and could potentially shed light on the current finding. It is important to note that all patients in this study were physically well recovered, evident from their upper limb clinical scores (grip and NHPT, Table 1). Therefore, we did not expect there to be an effect of hemiparetic side on their ability to perform the task successfully.

\section{Mechanism driving the change in trait fatigue}

One of the aims of this work was to identify the potential mechanisms that underlie the reduction in trait fatigue following anodal tDCS. The expectation was that a change immediately post stimulation in M1 neurophysiology and $\mathrm{PE}$ will result in a reduction in trait fatigue a week later. However, no change immediately post stimulation, but only a week later in these measures, makes it harder to interpret the neurophysiology and perception results. The results of the multiple linear regression model suggest that measures of M1 neurophysiology, specifically of the affected hemisphere a week after stimulation, appear to be related to the improving fatigue symptoms. Whether the change in fatigue is a consequence of a change in neurophysiology or whether they occur via independent mechanisms cannot be inferred from the current study. Therefore, from a mechanistic point of view, there are still questions to be addressed with regards to how anodal tDCS results in reduced trait fatigue. The improvement in fatigue symptoms does however appear to be modulated by anxiety levels prior to the stimulation itself. PSF and anxiety co-occur more often than any other problems such as pain, depression and sleep [64]. In chronic pain, anxiety exacerbates pain [65]. Similarly, anxiety levels may exacerbate fatigue which manifests as a small or no change in fatigue in those with high baseline anxiety. Anxiolytic medication (e.g. diazepam, lorazepam) targets the neurotransmitter GABA and anodal tDCS enhances cortical excitability by decreasing GABA concentration [66]. This could have prevented the efficacy of tDCS in reducing fatigue, however, patients included in the current study were not on any centrally acting medication. Therefore, the true interaction between anxiety and effect of tDCS remains unclear.

\section{Limitations}

Despite providing a potential intervention to improve fatigue symptoms after stroke, this study is not without limitations. Firstly, the current study is limited to non-depressed, minimally impaired stroke survivors. Given the nature of the symptom being investigated and the heterogeneous cohort that are stroke survivors, the effect of anodal tDCS on poststroke fatigue should be investigated in a wider range of stroke survivors. Secondly, due to the nature of the symptom being investigated, a small number of trials was used for the recruitment curve data and only a single session of anodal tDCS was performed to ensure that all patients could complete the study. Having multiple sessions of tDCS as in previous studies in multiple sclerosis [31, 33-37, 67, 68] might result in improvement in fatigue scores lasting longer than a week. Finally, despite differences in neurophysiological and PE measures between the real and sham stimulation groups, the reduction in fatigue scores is not fully explained 
by these measures, leaving the question of the mechanism of tDCS-induced reduction in fatigue, still open.

\section{Conclusion}

Our results show that a single session of bilateral anodal tDCS over the primary motor cortex improves fatigue symptoms for up to a week after stimulation. Therefore, tDCS could be a useful addition for the management of PSF. For effective interventions to be developed, we must improve our understanding of the structural and functional neural networks associated with altered effort perception, neurophysiological variables and PSF.

\section{Data availability statement}

The data that support the findings of this study are available from the corresponding author upon reasonable request.

Acknowledgements We sincerely thank Profs Nick Ward and John Rothwell for the many useful discussions and encouragement throughout the project. We thank Mr Cameron Cook and the Clinical Research Network for their help with recruitment. We thank our lab manager $\mathrm{Mr}$ Paul Hammond for the technical support throughout the project. We extend our heartfelt thanks to all our stroke survivor participants in this study without whose enthusiasm and commitment this study would not have been possible.

Author contributions Conceptualization: AK, SO; Methodology: AK, SO, WDD; Formal analysis and investigation: AK, SO, WDD; Writing-original draft preparation: WDD; Writing-review and editing: AK, SO, WDD; Funding acquisition: AK; Supervision: AK.

Funding This work was supported by the Wellcome Trust (202346/Z/16/Z) and Stroke Association (SA2015/02).

\section{Compliance with ethical standards}

Conflicts of interest The authors declare that they have no conflict of interest.

Ethical standards This study was performed in line with the principles of the Declaration of Helsinki. Approval was granted by the London Bromley Reasearch Ethics Committee (16/LO/0714).

Open Access This article is licensed under a Creative Commons Attribution 4.0 International License, which permits use, sharing, adaptation, distribution and reproduction in any medium or format, as long as you give appropriate credit to the original author(s) and the source, provide a link to the Creative Commons licence, and indicate if changes were made. The images or other third party material in this article are included in the article's Creative Commons licence, unless indicated otherwise in a credit line to the material. If material is not included in the article's Creative Commons licence and your intended use is not permitted by statutory regulation or exceeds the permitted use, you will need to obtain permission directly from the copyright holder. To view a copy of this licence, visit http://creativecommons.org/licenses/by/4.0/.

\section{References}

1. Cumming TB, Packer M, Kramer SF, English C (2016) The prevalence of fatigue after stroke: a systematic review and metaanalysis. Int J Stroke 11:968-977. https://doi.org/10.1177/17474 93016669861

2. Glader E-L, Stegmayr B, Asplund K (2002) Poststroke fatigue: a 2-year follow-up study of stroke patients in Sweden. Stroke 33:1327-1333

3. Christopher MK, Nina F, Judith R et al (2011) Self-reported long-term needs after stroke. Stroke 42:1398-1403. https://doi. org/10.1161/STROKEAHA.110.598839

4. Rudberg A-S, Berge E, Laska A-C et al (2020) Stroke survivors' priorities for research related to life after stroke. Top Stroke Rehabil. https://doi.org/10.1080/10749357.2020.1789829

5. Pollock A, St George B, Fenton M, Firkins L (2014) Top 10 research priorities relating to life after stroke consensus from stroke survivors, caregivers, and health professionals. Int J Stroke 9:313-320. https://doi.org/10.1111/j.1747-4949.2012.00942.x

6. Wu S, Kutlubaev MA, Chun H-YY et al (2015) Interventions for post-stroke fatigue. Cochrane Database Syst Rev. https://doi. org/10.1002/14651858.CD007030.pub3

7. Kuppuswamy A, Clark EV, Turner IF et al (2015) Post-stroke fatigue: a deficit in corticomotor excitability? Brain 138:136-148. https://doi.org/10.1093/brain/awu306

8. Rosso C, Lamy J-C (2018) Does resting motor threshold predict motor hand recovery after stroke? Front Neurol 9:1020. https:// doi.org/10.3389/fneur.2018.01020

9. Voss M, Bays PM, Rothwell JC, Wolpert DM (2007) An improvement in perception of self-generated tactile stimuli following theta-burst stimulation of primary motor cortex. Neuropsychologia 45:2712-2717. https://doi.org/10.1016/j.neuropsychologi a.2007.04.008

10. Ruge D, Muggleton N, Hoad D et al (2014) An unavoidable modulation? Sensory attention and human primary motor cortex excitability. Eur J Neurosci 40:2850-2858. https://doi.org/10.1111/ ejn. 12651

11. Zénon A, Sidibé M, Olivier E (2015) Disrupting the supplementary motor area makes physical effort appear less effortful. J Neurosci 35:8737-8744. https://doi.org/10.1523/JNEUR OSCI.3789-14.2015

12. Marcora S (2009) Perception of effort during exercise is independent of afferent feedback from skeletal muscles, heart, and lungs. J Appl Physiol 106:2060-2062. https://doi.org/10.1152/japplphysi ol.90378.2008

13. de Morree HM, Klein C, Marcora SM (2012) Perception of effort reflects central motor command during movement execution. Psychophysiology 49:1242-1253. https://doi.org/10.111 1/j.1469-8986.2012.01399.x

14. Slobounov S, Hallett M, Newell KM (2004) Perceived effort in force production as reflected in motor-related cortical potentials. Clin Neurophysiol 115:2391-2402. https://doi.org/10.1016/j.clinp h.2004.05.021

15. Lafargue G, Paillard J, Lamarre Y, Sirigu A (2003) Production and perception of grip force without proprioception: is there a sense of effort in deafferented subjects? Eur J Neurosci 17:2741-2749

16. Scotland S, Adamo DE, Martin BJ (2014) Sense of effort revisited: relative contributions of sensory feedback and efferent copy. Neurosci Lett 561:208-212. https://doi.org/10.1016/j.neule t.2013.12.041 
17. Gandevia SC (1982) The perception of motor commands or effort during muscular paralysis. Brain 105:151-159

18. Seth AK, Friston KJ (2016) Active interoceptive inference and the emotional brain. Philos Trans R Soc Lond B Biol Sci. https://doi. org/10.1098/rstb.2016.0007

19. Brown H, Adams RA, Parees I et al (2013) Active inference, sensory attenuation and illusions. Cogn Process 14:411-427. https:// doi.org/10.1007/s10339-013-0571-3

20. Kuppuswamy A (2017) The fatigue conundrum. Brain 140:22402245. https://doi.org/10.1093/brain/awx153

21. Doncker WD, Charles L, Ondobaka S, Kuppuswamy A (2020) Exploring the relationship between effort perception and poststroke fatigue. Neurology 95:e3321-e3330. https://doi. org/10.1212/WNL.0000000000010985

22. Nitsche MA, Paulus W (2000) Excitability changes induced in the human motor cortex by weak transcranial direct current stimulation. J Physiol 527:633-639. https://doi. org/10.1111/j.1469-7793.2000.t01-1-00633.x

23. Stagg CJ, Nitsche MA (2011) Physiological basis of transcranial direct current stimulation. Neuroscientist 17:37-53. https://doi. org/10.1177/1073858410386614

24. Brunoni AR, Nitsche MA, Bolognini N et al (2012) Clinical research with transcranial direct current stimulation (tDCS): challenges and future directions. Brain Stimul 5:175-195. https ://doi.org/10.1016/j.brs.2011.03.002

25. Fregni F, Nitsche MA, Loo CK et al (2015) Regulatory considerations for the clinical and research use of transcranial direct current stimulation (tDCS): review and recommendations from an expert panel. Clin Res Regul Aff 32:22-35. https://doi. org/10.3109/10601333.2015.980944

26. Bastani A, Jaberzadeh S (2014) Within-session repeated a-tDCS: the effects of repetition rate and inter-stimulus interval on corticospinal excitability and motor performance. Clin Neurophysiol 125:1809-1818. https://doi.org/10.1016/j.clinp h.2014.01.010

27. Nitsche MA, Cohen LG, Wassermann EM et al (2008) Transcranial direct current stimulation: state of the art 2008. Brain Stimul 1:206-223. https://doi.org/10.1016/j.brs.2008.06.004

28. Bastani A, Jaberzadeh S (2012) Does anodal transcranial direct current stimulation enhance excitability of the motor cortex and motor function in healthy individuals and subjects with stroke: a systematic review and meta-analysis. Clin Neurophysiol 123:644657. https://doi.org/10.1016/j.clinph.2011.08.029

29. Nitsche MA, Boggio PS, Fregni F, Pascual-Leone A (2009) Treatment of depression with transcranial direct current stimulation (tDCS): a review. Exp Neurol 219:14-19. https://doi. org/10.1016/j.expneurol.2009.03.038

30. Kuo M-F, Paulus W, Nitsche MA (2014) Therapeutic effects of non-invasive brain stimulation with direct currents (tDCS) in neuropsychiatric diseases. NeuroImage 85:948-960. https://doi. org/10.1016/j.neuroimage.2013.05.117

31. Saiote $C$, Goldschmidt $T$, Timäus $C$ et al (2014) Impact of transcranial direct current stimulation on fatigue in multiple sclerosis. Restor Neurol Neurosci 32:423-436. https://doi.org/10.3233/ RNN-130372

32. Chalah MA, Riachi N, Ahdab R et al (2015) Fatigue in multiple sclerosis: neural correlates and the role of non-invasive brain stimulation. Front Cell Neurosci. https://doi.org/10.3389/fncel .2015 .00460

33. Charvet LE, Dobbs B, Shaw MT et al (2018) Remotely supervised transcranial direct current stimulation for the treatment of fatigue in multiple sclerosis: Results from a randomized, sham-controlled trial. Mult Scler 24:1760-1769. https://doi.org/10.1177/13524 58517732842

34. Ferrucci R, Vergari M, Cogiamanian F et al (2014) Transcranial direct current stimulation (tDCS) for fatigue in multiple sclerosis.
Neuro Rehabilit 34:121-127. https://doi.org/10.3233/NRE-13101 9

35. Tecchio F, Cancelli A, Cottone C et al (2014) Multiple sclerosis fatigue relief by bilateral somatosensory cortex neuromodulation. J Neurol 261:1552-1558. https://doi.org/10.1007/s0041 5-014-7377-9

36. Ayache SS, Palm U, Chalah MA et al (2016) Prefrontal tDCS decreases pain in patients with multiple sclerosis. Front Neurosci. https://doi.org/10.3389/fnins.2016.00147

37. Tecchio F, Cancelli A, Cottone C et al (2015) Brain plasticity effects of neuromodulation against multiple sclerosis fatigue. Front Neurol. https://doi.org/10.3389/fneur.2015.00141

38. Angius L, Mauger AR, Hopker J et al (2018) Bilateral extracephalic transcranial direct current stimulation improves endurance performance in healthy individuals. Brain Stimul 11:108-117. https://doi.org/10.1016/j.brs.2017.09.017

39. Valko PO, Bassetti CL, Bloch KE et al (2008) Validation of the Fatigue Severity Scale in a Swiss Cohort. Sleep 31:1601-1607

40. Nordin A, Taft C, Lundgren-Nilsson A, Dencker A (2016) Minimal important differences for fatigue patient reported outcome measures-a systematic review. BMC Med Res Methodol. https ://doi.org/10.1186/s12874-016-0167-6

41. Rooney S, McFadyen DA, Wood DL et al (2019) Minimally important difference of the fatigue severity scale and modified fatigue impact scale in people with multiple sclerosis. Mult Scler Relat Disord 35:158-163. https://doi.org/10.1016/j.msard .2019.07.028

42. Wiethoff S, Hamada M, Rothwell JC (2014) Variability in response to transcranial direct current stimulation of the motor cortex. Brain Stimul 7:468-475. https://doi.org/10.1016/j. brs.2014.02.003

43. Krupp LB, LaRocca NG, Muir-Nash J, Steinberg AD (1989) The fatigue severity scale: application to patients with multiple sclerosis and systemic lupus erythematosus. Arch Neurol 46:11211123. https://doi.org/10.1001/archneur.1989.00520460115022

44. Snaith RP (2003) The hospital Anxiety And Depression Scale. Health Qual Life Outcomes 1:29. https://doi. org/10.1186/1477-7525-1-29

45. Bikson M, Grossman P, Thomas C et al (2016) Safety of transcranial direct current stimulation: evidence based update 2016. Brain Stimulation 9:641-661. https://doi.org/10.1016/j.brs.2016.06.004

46. Ambrus GG, Al-Moyed H, Chaieb L et al (2012) The fade-inshort stimulation-fade out approach to sham tDCS-reliable at $1 \mathrm{~mA}$ for naïve and experienced subjects, but not investigators. Brain Stimul 5:499-504. https://doi.org/10.1016/j.brs.2011.12.001

47. Lang N, Siebner HR, Ward NS et al (2005) How does transcranial DC stimulation of the primary motor cortex alter regional neuronal activity in the human brain? Eur J Neurosci 22:495-504. https://doi.org/10.1111/j.1460-9568.2005.04233.x

48. de Morree HM, Klein C, Marcora SM (2014) Cortical substrates of the effects of caffeine and time-on-task on perception of effort. J Appl Physiol. https://doi.org/10.1152/japplphysiol.00898.2013

49. Hillis AE (2020) Developments in treating the nonmotor symptoms of stroke. Expert Rev Neurother 20:567-576. https://doi. org/10.1080/14737175.2020.1763173

50. Aali G, Drummond A, de Nair R, Shokraneh F (2020) Poststroke fatigue: a scoping review. F1000Res 9:242. https://doi. org/10.12688/f1000research.22880.1

51. Karabanov A, Ziemann U, Hamada M et al (2015) Consensus paper: probing homeostatic plasticity of human cortex with noninvasive transcranial brain stimulation. Brain Stimul 8:442-454. https://doi.org/10.1016/j.brs.2015.01.404

52. Chen K-H, Huang Y-Z (2018) The change of motor cortical excitability between eyes open and closed conditions. NeuroReport 29:214-218. https://doi.org/10.1097/WNR.0000000000000955 
53. Potter-Baker KA, Varnerin NM, Cunningham DA et al (2016) Influence of corticospinal tracts from higher order motor cortices on recruitment curve properties in stroke. Front Neurosci 10:79. https://doi.org/10.3389/fnins.2016.00079

54. Ridding MC, Rothwell JC (1997) Stimulus/response curves as a method of measuring motor cortical excitability in man. Electroencephalogr Clin Neurophysiol 105:340-344. https://doi. org/10.1016/s0924-980x(97)00041-6

55. Ward NS, Newton JM, Swayne OBC et al (2006) Motor system activation after subcortical stroke depends on corticospinal system integrity. Brain 129:809-819. https://doi.org/10.1093/brain/awl00 2

56. Smith MR, Coutts AJ, Merlini M et al (2016) Mental fatigue impairs soccer-specific physical and technical performance. Med Sci Sports Exerc 48:267-276. https://doi.org/10.1249/MSS.00000 00000000762

57. Van Cutsem J, Marcora S, De Pauw K et al (2017) The effects of mental fatigue on physical performance: a systematic review. Sports Med. https://doi.org/10.1007/s40279-016-0672-0

58. Jones PR, Moore DR, Shub DE, Amitay S (2015) The role of response bias in perceptual learning. J Exp Psychol Learn Mem Cogn 41:1456-1470. https://doi.org/10.1037/xlm0000111

59. Moore TM, Picou EM (2018) A potential bias in subjective ratings of mental effort. J Speech Lang Hear Res 61:2405-2421. https:// doi.org/10.1044/2018_JSLHR-H-17-0451

60. Crosby GA, Munshi S, Karat AS et al (2012) Fatigue after stroke: frequency and effect on daily life. Disabil Rehabil 34:633-637. https://doi.org/10.3109/09638288.2011.613517

61. Walsh ME, Galvin R, Loughnane $\mathrm{C}$ et al (2015) Factors associated with community reintegration in the first year after stroke: a qualitative meta-synthesis. Disabil Rehabil 37:1599-1608. https ://doi.org/10.3109/09638288.2014.974834

62. Proffitt DR, Stefanucci J, Banton T, Epstein W (2003) The role of effort in perceiving distance. Psychol Sci 14:106-112. https:// doi.org/10.1111/1467-9280.t01-1-01427

63. Clark EV, Ward NS, Kuppuswamy A (2016) Prior physical exertion modulates allocentric distance perception: a demonstration of task-irrelevant cross-modal transfer. Exp Brain Res 234:23632367. https://doi.org/10.1007/s00221-016-4641-5

64. Naess H, Lunde L, Brogger J, Waje-Andreassen U (2012) Fatigue among stroke patients on long-term follow-up. The Bergen Stroke Study. J Neurol Sci 312:138-141. https://doi.org/10.1016/j. jns.2011.08.002

65. Ploghaus A, Narain C, Beckmann CF et al (2001) Exacerbation of pain by anxiety is associated with activity in a hippocampal network. J Neurosci 21:9896-9903. https://doi.org/10.1523/JNEUR OSCI.21-24-09896.2001

66. Antonenko D, Schubert F, Bohm F et al (2017) tDCS-induced modulation of GABA levels and resting-state functional connectivity in older adults. J Neurosci 37:4065-4073. https://doi. org/10.1523/JNEUROSCI.0079-17.2017

67. Chalah MA, Grigorescu C, Padberg F et al (2020) Bifrontal transcranial direct current stimulation modulates fatigue in multiple sclerosis: a randomized sham-controlled study. J Neural Transm 127:953-961. https://doi.org/10.1007/s00702-020-02166-2

68. Cancelli A, Cottone C, Giordani A et al (2018) Personalized, bilateral whole-body somatosensory cortex stimulation to relieve fatigue in multiple sclerosis. Mult Scler 24:1366-1374. https:// doi.org/10.1177/1352458517720528 